\title{
SPLIT EXACT SEQUENCES OF FINITE MTL-CHAINS
}

\author{
J. L. CASTIGLIONI AND W. J. ZULUAGA BOTERO
}

\begin{abstract}
This paper is devoted to presenting ordinal sums of MTL-chains as a particular case of split short exact sequences of finite chains in the category of semihoops. This module theoretical approach will allows us to prove, in a very elementary way, that every finite locally unital MTL-chain can be decomposed as an ordinal sum of archimedean MTL-chains. Furthermore, we propose the study of MTL-chain extensions and we show that ordinal sums of locally unital MTL-chains are a particular case of these.
\end{abstract}

\section{INTRODUCTION}

In [6], Esteva and Godo proposed a new Hilbert-style calculus called monoidal t-norm based logic (MTL, for short) in order to find the fuzzy logic corresponding to the class of all left-continuous t-norms. In [8], Jenei and Montagna proved that MTL was, in fact, the weakest logic which is complete with respect to a semantics given by a class of t-norms and their residua. Since in MTL the contraction rule does not hold in general, such a logic can be regarded not only as a fuzzy logic and as a many-valued logic, but also as a substructural logic. These results motivated the introduction of a new class of algebras with an equivalent algebraic semantics for MTL, the variety of MTL-algebras. MTL-algebras are essentially integral commutative residuated lattices with bottom satisfying the prelinearity equation

$$
(x \rightarrow y) \vee(y \rightarrow x) \approx 1 .
$$

In [4], Castiglioni and Zuluaga characterized the class of finite MTL-chains which can be decomposed as an ordinal sum of archimedean MTL-chains. Such a class of finite MTL-algebras was called locally unital. Nevertheless, the general problem of decompositions by ordinal sums of (arbitrary) MTL-chains still remains open. It is worth mentioning that the general problem was already solved for the subvariety of BL-chains (see [1, 2]) by proving that (arbitrary) BL-chains can be decomposed as an ordinal sum of Wajsberg hoops.

Since its foundation, algebraic logic has been devoted to studying logics from the perspective of universal algebra. But it is important to recall that there are some works in the literature where starting from algebraic structures which are closer to rings - results of interest in algebraic logic have been obtained (see [3]).

2020 Mathematics Subject Classification. 03G27, 06D99. 
These facts bring out the question of the potential of employing tools of classical algebra (rings, modules, etc.) in order to study algebraic structures associated to non classical logics.

Inspired in extensions of rings and modules and their relation with ordinal sums, we intend to apply such methods to the context of finite MTL-chains. We will show that this approach allows us to prove results like the decomposition of locally unital MTL-chains as an ordinal sum of archimedean MTL-chains (obtained in [4]) in a very self-contained and elementary way, by applying ideas of split short exact sequences of finite chains in the category of semihoops. Additionally, we propose a construction called MTL-chain extension and we prove that such a construction turns out to be a suitable generalization of ordinal sums for locally unital MTLchains.

The first section is devoted to introducing all the concepts required to read this work. In the second section we obtain a characterization of those extensions of finite totally ordered semihoops which are isomorphic to an ordinal sum. In the third section we show that every finite locally unital MTL-chain can be decomposed as the ordinal sum of archimedean MTL-chains. Finally, in the last section, we introduce the MTL-chain extensions and we prove that the ordinal sums of finite totally ordered semihoops are a particular case of these.

\section{Preliminaries}

A semihoop $]^{1}[7$ is an algebra $\mathbf{A}=(A, \cdot, \rightarrow, \wedge, \vee, 1)$ of type $(2,2,2,2,0)$ such that $(A, \wedge, \vee)$ is a lattice with 1 as greatest element, $(A, \cdot, 1)$ is a commutative monoid and for every $x, y, z \in A$ the following conditions hold:

$$
\begin{array}{ll}
\text { (residuation) } & x y \leq z \text { if and only if } x \leq y \rightarrow z \\
\text { (prelinearity) } & (x \rightarrow y) \vee(y \rightarrow x)=1 .
\end{array}
$$

Equivalently, a semihoop is an integral, commutative and prelinear residuated lattice. We write $\mathcal{S H}$ for the algebraic category of semihoops. A semihoop $\mathbf{A}$ is bounded if $(A, \wedge, \vee, 1)$ has a least element 0 . An $M T L$-algebra is a bounded semihoop; hence, MTL-algebras are prelinear integral bounded commutative residuated lattices, as usually defined [6, 10], and semihoops are basically "MTL-algebras without zero". An MTL-algebra (or semihoop) A is an MTL-chain (SH-chain) if its semihoop reduct is totally ordered. It is a well-known fact that the class of MTL-algebras is a variety. We write $\mathcal{M T} \mathcal{L}$ for the category of MTL-algebras and MTL-homomorphisms. A totally ordered MTL-algebra is archimedean if for every $x \leq y<1$, there exists $n \in \mathbb{N}$ such that $y^{n} \leq x$. A submultiplicative monoid $F$ of $M$ is called a filter if it is an up-set with respect to the order of $M$. For every $x \in F$, we write $\langle x\rangle$ for the filter generated by $x$; i.e.,

$$
\langle x\rangle=\left\{a \in M \mid x^{n} \leq a \text { for some } n \in \mathbb{N}\right\} .
$$

For any filter $F$ of $M$, we can define the relation $\sim_{F}$ on $M$ by $a \sim_{F} b$ if and only if $a \rightarrow b \in F$ and $b \rightarrow a \in F$. It follows that $\sim_{F}$ is indeed a congruence on $M$.

\footnotetext{
${ }^{1}$ Also called basic semihoop in [1].
} 
On MTL-algebras there exists a well-known correspondence between filters and congruences (see [10]) so we write the quotient $M / \sim_{F}$ by $M / F$. For every $a \in M$, we write $[a]_{F}$ for the equivalence class of $a$ in $M / F$. If there is no ambiguity, we simply write $[a]$. A filter $F$ of $M$ is prime if $0 \notin F$ and $x \vee y \in F$ entails $x \in F$ or $y \in F$, for every $x, y \in M$.

Let $\mathbf{I}=(I, \leq)$ be a totally ordered set and $\mathcal{F}=\left\{\mathbf{A}_{i}\right\}_{i \in I}$ a family of semihoops. Let us assume that the members of $\mathcal{F}$ share (up to isomorphism) the same neutral element; i.e., for every $i \neq j, A_{i} \cap A_{j}=\{1\}$. The ordinal sum of the family $\mathcal{F}$ is the structure $\bigoplus_{i \in I} A_{i}$ whose universe is $\bigcup_{i \in I} A_{i}$ and whose operations are defined as

$$
\begin{gathered}
x \cdot y= \begin{cases}x \cdot_{i} y, & \text { if } x, y \in A_{i}, \\
y, & \text { if } x \in A_{i} \text { and } y \in A_{j}-\{1\}, \text { with } i>j, \\
x, & \text { if } x \in A_{i}-\{1\} \text { and } y \in A_{j}, \text { with } i<j ;\end{cases} \\
x \rightarrow y= \begin{cases}x \rightarrow_{i} y, & \text { if } x, y \in A_{i}, \\
y, & \text { if } x \in A_{i} \text { and } y \in A_{j}, \text { with } i>j, \\
1, & \text { if } x \in A_{i}-\{1\} \text { and } y \in A_{j}, \text { with } i<j,\end{cases}
\end{gathered}
$$

where the subindex $i$ denotes the operations in $A_{i}$.

Furthermore, if $\mathbf{I}$ has a minimum $\perp, A_{i}$ is a totally ordered semihoop for every $i \in \mathbf{I}$ and $A_{\perp}$ is bounded, then $\bigoplus_{i \in I} A_{i}$ becomes an MTL-chain. In order to clarify notation, we will use the symbol $\boxplus$ to denote the usual linear sum of lattices (as defined in [5, Section 1.24]).

Let $M$ be an MTL-algebra. Write 0,1 for the trivial idempotents, $\mathcal{I}(M)$ for the set of all idempotent elements of $M$ and $\mathcal{I}^{*}(M)$ for $\mathcal{I}(M)-\{0\}$. We say that $e \in \mathcal{I}^{*}(M)$ is a local unit if $x e=x$, for all $x \leq e$. Clearly, 1 is a local unit. If $M$ is archimedean, 1 is in fact the only local unit of $M$. Notice that there may be idempotents which are not local units. As an example, one may consider the MTL-algebra A whose underlying set is the totally ordered set $A=\{0, x, e, 1\}$, where $0<x<e<1$, with the product determined by the following table:

\begin{tabular}{c|c|c|c|c|}
$\cdot$ & 1 & $e$ & $x$ & 0 \\
\hline 1 & 1 & $e$ & $x$ & 0 \\
\hline$e$ & $e$ & $e$ & 0 & 0 \\
\hline$x$ & $x$ & 0 & 0 & 0 \\
\hline 0 & 0 & 0 & 0 & 0 \\
\hline
\end{tabular}

TABLE 1. MTL algebra with an idempotent which is not a local unit.

In 4], the class of MTL-algebras whose non-trivial idempotents are exactly the local units was called locally unital MTL-algebras (luMTL, for short). In this

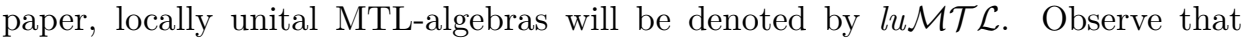
every archimedean chain is a member of $l u \mathcal{M T} \mathcal{L}$. 


\section{Another PERSPECTIVE ON FINITE LUMTL-ChAins}

We start this section with the following observation: Let $M$ be a semihoop and $F$ a filter of $M$. A straightforward verification shows that $F$ is a subalgebra of $M$, which basically says that in $\mathcal{S H}$, filters always belong to the same category of the original algebra. Such a situation does not hold for MTL-algebras, since the only filter of $M$ which is a subalgebra is $M$ itself. Hence, taking advantage of the fact that MTL-algebras are "semihoops with zero", along this section we will place ourselves in the context of semihoops, rather than that of MTL-algebras.

In $\mathcal{S H}$ a sequence of morphisms

$$
\mathbf{0} \longrightarrow A \stackrel{f}{\longrightarrow} B \stackrel{g}{\longrightarrow} C \longrightarrow \mathbf{0}
$$

is a short exact sequence if $f$ is injective and $g$ is surjective. Here $\mathbf{0}=\{1\}$ is the zero object of the category. The short exact sequence is a split exact sequence if $g$ has a section, i.e., if there exists $h: C \rightarrow B$ such that $g h=i d_{C}$.

An interesting problem is to classify all the split exact sequences in $\mathcal{S H}$. Nevertheless, in this paper we give a solution for a more modest problem. The aim of this section is to present a characterization of the split exact sequences in which $C$ is a chain and $B$ is isomorphic to the ordinal sum of $C$ with $A$.

Lemma 2.1. Let $K, E, C \in \mathcal{S H}$, where $C$ is a chain. Consider the split exact sequence

$$
\mathbf{0} \longrightarrow K \stackrel{k}{\longrightarrow} E \stackrel{s}{\stackrel{\leftrightarrow}{\longrightarrow}} C \longrightarrow \mathbf{0} .
$$

Then, $E \cong C \oplus K$ if and only if

i) $E=k(K) \cup s(C)$ and

ii) $k(K) \cap s(C)=\mathbf{0}$.

Proof. Let us assume that the split exact sequence above satisfies (i) and (ii). Since $s, k$ are injective morphisms of semihoops, $s(C)$ and $k(K)$ are subobjects of $E$ isomorphic to $K$ and $C$, respectively. So let us simply write $K$ for $k(K)$ and $C$ for $s(C)$. Let $e \in E$. By (i), $e \in K$ or $e \in C$. If $e \in K \cap C$, then, by (ii), $e=1$. If $e \in K$ and $f \in E$ is such that $e \leq f$, then from $1=p(e) \leq p(f)$ we get that $p(f)=1$. Hence $f \in K$. Consequently, $K$ is a filter in $E$, and every $c \in C-\{1\}$ is below every $k \in K$. Therefore, as a lattice, $E \cong(C-\{1\}) \boxplus K$. Notice that both $K$ and $C$ are closed by the product and the residuum. So we just have to calculate $c k, c \rightarrow k$ and $k \rightarrow c$ for $c \in C$ and $k \in K$. Observe that we can assume $c \neq 1$. From the calculation

$$
p(c k)=p(c) p(k)=p(c) \cdot 1=p(c)
$$

we can conclude that $c k=c$ for every $c \in C$ and $k \in K$. Taking into account the order of $E$, we obtain $c k=c \wedge k$. On the other hand, $p(k \rightarrow c)=1 \rightarrow p(c)=p(c)$ so $k \rightarrow c=c$; and $p(c \rightarrow k)=p(c) \rightarrow 1=1$, hence $c \rightarrow k \in K$. Finally, since $c \wedge k^{\prime}=c k^{\prime} \leq c \leq k$ for every $k^{\prime} \in K$, we have $c \rightarrow k=1$. 
We have proved that the binary operations on $E \cong(C-\{1\}) \boxplus K$ are given by:

$$
\begin{aligned}
& k c= \begin{cases}k \cdot_{K} c, & k, c \in K, \\
k \cdot C c, & k, c \in C, \\
k \wedge c, & \text { otherwise; }\end{cases} \\
& k \rightarrow c= \begin{cases}k \rightarrow_{K} c, & k, c \in K, \\
k \rightarrow_{C} c, & k, c \in C, \\
c, & k \in K, c \in C, \\
1, & \text { otherwise. }\end{cases}
\end{aligned}
$$

That is to say, $E \cong K \oplus C$, the ordinal sum of semihoops. The converse follows directly from the definition of ordinal sum of semihoops.

To conclude this section we would like to remark that in $\mathcal{S H}$ there are split exact sequences which are not of the kind presented in Lemma 2.1. In order to exhibit one, consider the semihoop $A=\{0, x, e, 1\}$ whose product is defined in Table 1 Let $F=\uparrow e$ and let $\mathbf{2}$ be the chain of two elements. It is clear that $A / F \cong \mathbf{2}$ and that $s: \mathbf{2} \rightarrow A$, defined by $s(1)=1_{A}$ and $s(0)=0_{A}$, is a section of the quotient map, so $\mathbf{0} \rightarrow F \rightarrow A \rightarrow \mathbf{2} \rightarrow \mathbf{0}$ is a split exact sequence. Nevertheless, $x \notin s(\mathbf{2}) \cup k(F)$ so $A \not \mathbf{2} \oplus F$.

\section{Dealing with Finite Chains in LUMTL}

In [1, Aglianò and Montagna proved that every BL-chain can be decomposed as an ordinal sum of totally ordered Wajsberg hoops. In [2, Busaniche provides an alternative proof of this fact without using the axiom of choice. In 4, it was observed that, as a consequence of the divisibility condition for BL-algebras ${ }^{2}$ it follows that every BL-algebra is a locally unital MTL-algebra. The aim of this section is to generalize the finite case of Aglianò and Montagna's result to finite locally unital MTL-chains. That is to say, to give, through the tools developed along this work, an elementary proof of the fact that every finite locally unital MTL-chain decomposes as an ordinal sum of archimedean MTL-chains (see 4 for another proof).

Let $M$ be a finite MTL-chain (or SH-chain). The following useful characterization can be found in [4.

Proposition 3.1. Let $M$ be a finite MTL-chain. The following are equivalent:

i) $M$ is archimedean,

ii) $M$ is simple,

iii) $\mathcal{I}(M)=\{0,1\}$.

Write $\langle X\rangle$ for the filter generated by $X \subseteq M$ and $\mathcal{L U}(M)$ for the set of local units of $M$. Note that in general $\mathcal{L U}(M) \subseteq \mathcal{I}^{*}(M)$. Furthermore, $M \in \operatorname{lu} \mathcal{M T} \mathcal{L}$ if and only if $\mathcal{L U}(M)=\mathcal{I}^{*}(M)$.

\footnotetext{
${ }^{2}$ That is, for every $x, y$ the equation $x(x \rightarrow y)=x \wedge y$ holds.
} 
Proposition 3.2 ([4, Corollary 4]). In any finite MTL-algebra $M$, the following are equivalent:

i) $a \in \mathcal{I}(M)$,

ii) $\langle a\rangle=\uparrow a$.

Note that if $M$ is finite and $F$ is a proper filter of $M$, there exists a unique $e \in \mathcal{I}^{*}(M)$ such that $F=\uparrow e$. We write $e M$ for the set $e M=\{e x \mid x \in M\}$.

Remark 3.3. Let $M$ be a finite MTL-chain and $e \in \mathcal{L U}(M)$. Then the sequence

$$
\mathbf{0} \longrightarrow \uparrow e \longrightarrow M \longrightarrow M / \uparrow e \longrightarrow \mathbf{0}
$$

is a split exact sequence of the type of Lemma 2.1 and $M / \uparrow e \cong e M$ (which is in fact true for any $e \in \mathcal{L U}(M))$. Hence $M \cong \uparrow e \oplus e M$.

Theorem 3.4. Every finite locally unital MTL-chain is an ordinal sum of archimedean chains.

Proof. Let $M$ be a finite locally unital MTL-chain. It follows that $\mathcal{L U}(M)$ is also a finite chain. In order to prove the statement, we proceed by induction over the amount of local units of $M$. If $|\mathcal{L U}(M)|=1$, then by Proposition $3.1 M$ is archimedean. Let us assume that $|\mathcal{L U}(M)|>1$ and let us order its elements in an increasing way:

$$
e_{1}<\ldots<e_{n}=1 .
$$

Let us suppose that any locally unital MTL-chain $A$ with $|\mathcal{L U}(A)|=n-1$ is an ordinal sum of $n-1$ archimedean chains:

$$
A \cong C_{1} \oplus \ldots \oplus C_{n-1} .
$$

Since $e_{n-1} \in \mathcal{L U}(M)$, by Remark 3.3 the sequence

$$
\mathbf{0} \longrightarrow \uparrow e_{n-1} \longrightarrow M \stackrel{s}{\stackrel{s}{\longrightarrow}} e_{n-1} M \longrightarrow \mathbf{0}
$$

is a split exact sequence of the type of Lemma 2.1. whence $M \cong \uparrow e_{n-1} \oplus e_{n-1} M$. Furthermore, $\uparrow e_{n-1}$ is archimedean by Proposition $\mid 3.1$ and $\left|\mathcal{L U}\left(e_{n-1} M\right)\right|=n-1$. By inductive hypothesis we can conclude that $e_{n-1} M \cong C_{1} \oplus \ldots \oplus C_{n-1}$. An easy verification shows that

$$
M \cong \uparrow e_{n-1} \oplus e_{n-1}\left(\uparrow e_{n-2}\right) \oplus \ldots \oplus e_{2}\left(\uparrow e_{1}\right) \oplus e_{1} M .
$$

This concludes the proof.

We conclude this section by presenting a characterization of some particular split exact sequences of the kind of those of Lemma 2.1.

Theorem 3.5. Let $M_{n}$ and $M_{n+1}$ be MTL-chains of $n$ and $n+1$ elements, respectively, for some $n>1$. Let $\alpha$ be the coatom of $M_{n+1}$. The following are equivalent:

(1) $\alpha$ is a local unit. 
(2) The sequence

$$
\mathbf{0} \longrightarrow \mathbf{2} \longrightarrow M_{n+1} \longrightarrow M_{n} \longrightarrow \mathbf{0}
$$

is a split exact sequence such that $M_{n+1} \cong M_{n} \oplus \mathbf{2}$.

Proof. Observe that the first implication is a straight consequence of Remark 3.3 So let us assume (2). Notice that as semihoops $\uparrow \alpha \cong \mathbf{2}$, then it follows that $\uparrow \alpha$ is a filter of $M_{n+1}$, so from Proposition 3.2 we obtain that $\alpha^{2}=\alpha$. Since $M_{n+1} \cong M_{n} \oplus \uparrow \alpha$, we have that as a poset $M_{n+1} \cong\left(M_{n}-\left\{1_{M_{n}}\right\}\right) \boxplus \uparrow \alpha$. Hence, if $x \in M_{n+1}$, since $\alpha$ is coatom, it is the case that $x<\alpha$, so $x \in M_{n}-\left\{1_{M_{n}}\right\}$ by hypothesis. Consequently, $x \cdot \alpha=x=x \wedge \alpha$. Therefore $\alpha$ is a local unit.

\section{A BRIEF DESCRIPTION OF MTL-CHAin EXTENSIONS}

In this section we propose a construction involving finite MTL-chains, namely, MTL-chain extensions. We will show that such a process is intimately related with split exact sequences involving totally ordered semihoops. Finally, we will show that such a construction can be regarded as a suitable generalization of ordinal sums of locally unital MTL-chains.

Let $F$ and $A$ be two finite MTL-chains. Since $A$ is finite, we can order its elements in an increasing way; let us say, $0_{A}<x_{1}<\ldots<x_{n-1}<1_{A}$. Let $\mathcal{C}_{(F, A)}=\left\{C_{j} \mid j \in A\right\}$ be a family of sets such that

(1) for every $i \in A, C_{i}$ is a finite chain;

(2) for $i=1_{A}, C_{1_{A}}=F$;

(3) if $i \neq j$, with $i, j \in A$, then $C_{i} \cap C_{j}=\emptyset$.

Let $E:=\bigcup_{i \in A} C_{i}$ with the order induced by the ordinal sum of lattices; that is, as a poset, $E=\boxplus_{i \in A} C_{i}$. Hence $E$ is a lattice. Observe that since each $C_{i}$ is a finite lattice it has a minimum element; let us write $0_{i}$ for it.

Now, take a family of functions $\mathbf{M}_{(F, A)}=\left\{\mu_{i j}: C_{i} \times C_{j} \rightarrow C_{i \cdot j} \mid i, j \in A\right\}$ (where $i \cdot j$ denotes the product in $A$ ), such that

(i) for every $i, j \in A, \mu_{i j}$ is monotone in each coordinate;

(ii) for every $k_{i} \in C_{i}$ and $k_{j} \in C_{j}, \mu_{i j}\left(0_{i}, k_{j}\right)=\mu_{i j}\left(k_{i}, 0_{j}\right)=0_{i j}$;

(iii) $\mathbf{M}_{(F, A)}$ is jointly associative; that is, the diagram

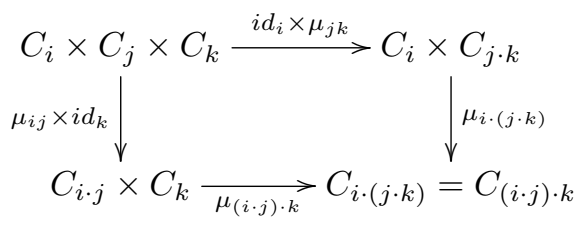

commutes, for every $i, j, k \in A$. 
(iv) $\mathbf{M}_{(F, A)}$ is jointly commutative; that is, the diagram

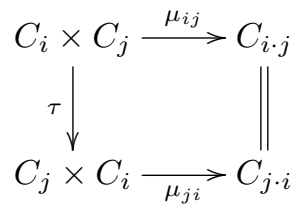

commutes, for every $i, j, k \in A$.

(v) $\mathbf{M}_{(F, A)}$ has a global unit; that is, the diagram

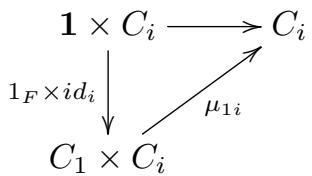

commutes, for every $i \in A$. Here $\mathbf{1}$ is the singleton chain and 1 is the unit of $A$.

This presentation leads us to introduce the following concept.

Definition 4.1. Let $F$ and $A$ be two finite MTL-chains. An MTL-chain extension (induced by $F$ and $A$ ) is a pair $\left(\mathcal{C}_{(F, A)}, \mathbf{M}_{(F, A)}\right)$ such that

1. the collection $\mathcal{C}_{(F, A)}=\left\{C_{j} \mid j \in A\right\}$ satisfies (1), (2) and (3);

2. the set $\mathbf{M}_{(F, A)}=\left\{\mu_{i j}: C_{i} \times C_{j} \rightarrow C_{i \cdot j} \mid i, j \in A\right\}$ is a family of functions satisfying (i) $-(\mathrm{v})$.

Theorem 4.2. Let $F$ and $A$ be two finite $M T L$-chains. Then, every $M T L$-chain extension $\left(\mathcal{C}_{(F, A)}, \mathbf{M}_{(F, A)}\right)$ induces a split exact sequence of $M T L$-chains.

Proof. Take $E\left(\mathcal{C}_{(F, A)}, \mathbf{M}_{(F, A)}\right):=\bigcup \mathcal{C}$ with the mentioned order. Endow $\mathbf{E}=$ $E\left(\mathcal{C}_{(F, A)}, \mathbf{M}_{(F, A)}\right)$ with the binary operation

$$
\mu: \mathbf{E} \times \mathbf{E} \rightarrow \mathbf{E}
$$

defined by $\mu(e, f)=\mu_{i j}(e, f)$, if $e \in C_{i}$ and $f \in C_{j}$. Observe that conditions (i) $-(\mathrm{v})$ of $\mathbf{M}$ guarantee that $\left(\mathbf{E}, \vee, \wedge, \mu, 1_{F}\right)$ is an MTL-algebra. Furthermore, $F=C_{1}$ is a filter of $\mathbf{E}$. Let us calculate $\mathbf{E} / F$. For $e, f \in \mathbf{E}, e \sim_{F} f$ if and only if there is a $g \in F$ such that $g e \leq f$ and $g f \leq e$, so it is clear that $F=[1]$. Now, let us assume $e, f \in C_{i}$ for some $i \neq 1_{A}$. From (ii), $\mu_{1_{A} i}\left(0_{1_{A}}, e\right)=\mu_{1_{A} i}\left(0_{1_{A}}, f\right)=0_{i}$ so $e \sim_{F} f$. If $e \in C_{i}$ and $f \in C_{j}$ for some $i \neq j$, from (3), $C_{i} \cap C_{j}=\emptyset$. Then for every $g \in F=C_{1_{A}}$ we have that $\mu_{1_{A} i}(g, e) \in C_{i}$ and $\mu_{1_{A} j} \in C_{j}$; that is, $e \nsim_{F} f$. Hence $C_{i}=[e]_{F}$ for any $e \in C_{i}$ and $i \in A$. So we have a bijection $\mathbf{E} / F \rightarrow A$ which clearly is an MTL-morphism. Moreover, the map $s: A \rightarrow \mathbf{E}$ defined by

$$
s(i)= \begin{cases}1_{F}, & i=1_{A}, \\ 0_{i}, & \text { otherwise }\end{cases}
$$


is a section of $p: \mathbf{E} \rightarrow A \cong \mathbf{E} / F$. Hence, we get a split short exact sequence in $\mathcal{S H}$ :

$$
\mathbf{0} \longrightarrow F \stackrel{j}{\longrightarrow} \mathbf{E} \stackrel{s}{\stackrel{s}{\longrightarrow}} A \longrightarrow \mathbf{0}
$$

where $j$ is the inclusion.

Let $(P, \leq)$ be a poset. A subset $X$ of $P$ is convex if for every $x \leq z \leq y$ such that $x, y \in X$ we have that $z \in X$. An ordered commutative monoid is a structure $\mathbf{S}=(S, \cdot, \leq, 1)$ such that $(S, \cdot, 1)$ is a commutative monoid, $(S, \leq)$ is a poset and is monotone with respect to $\leq$. If 1 is the top element of $(S, \leq)$, then $\mathbf{S}$ is called integra ${ }^{3}$ If $S$ is convex, then we say that $\mathbf{S}$ is an integral commutative convex monoid.

The following result is a partial converse of Lemma 4.2 .

Theorem 4.3. Let

$$
\mathbf{0} \longrightarrow F \stackrel{j}{\longrightarrow} \mathbf{E} \stackrel{p}{\longrightarrow} A \longrightarrow \mathbf{0}
$$

be a split exact sequence in $\mathcal{S H}$ such that

i) $j(F)$ is a convex subset of $E$ and

ii) $E, F$ and $A$ are finite chains.

Then, there exists a $M T L$-chain extension $\left(\mathcal{C}_{(F, A)}, \mathbf{M}_{(F, A)}\right)$ such that

$$
\mathbf{E} \cong E\left(\mathcal{C}_{(F, A)}, \mathbf{M}_{(F, A)}\right) \text {. }
$$

Proof. Observe that, since $j(F) \cong F$ is a convex submonoid of a finite integral ordered commutative monoid, $F$ is a filter of $E$ and hence there exists a unique idempotent $e_{F} \in E$ such that $F=\uparrow e_{F}$. For the rest of the proof we shall simply write $e$ for $e_{F}$. Notice that there is an isomorphism between $A$ and $e E$. By definition of short exact sequence, we know that $A$ is just $E / F$, so we can define a map $f: E / F \rightarrow e E$ by $f([a])=e a$ which is clearly bijective. From this fact we can conclude that $[a]=\{x \in E \mid e x=e a\}$. Now, since each equivalence class is a convex set of $E$ (because each class is in particular a lattice congruence class), we have a decomposition of $E$ as a lattice as $E \cong \boxplus_{[x] \in A} C_{[x]}$, where the $C_{[x]}$ 's form a partition of $E$ by convex chains. Clearly, this corresponds to the quotient map. Now take $\mathcal{C}=\{[a] \mid[a] \in A\}$ and define $\mu_{[a][b]}:[a] \times[b] \rightarrow[a b]$ by $\mu_{[a][b]}(\alpha, \beta)=\alpha \beta$, that is, the restriction of the product to the respective $C_{[x]}$ 's. It is easy to check that they form a family $\mathbf{M}$ satisfying conditions (i)-(v) of the beginning of this section.

Corollary 4.4. Ordinal sums of finite locally unital MTL-chains are MTL-chain extensions.

Proof. Let $\left\{M_{i}\right\}_{i \in I}$ be a family of finite locally unital MTL-chains, where $I$ is a finite totally ordered set with top $\top$. Take $\left\{M_{i}^{\prime}\right\}_{i \in I}$ with $M_{\top}^{\prime}=M_{\top}$ and $M_{i}^{\prime}=$ $M_{i}-\left\{1_{M_{i}}\right\}$ for $i \neq \top$. Note that each $M_{i}^{\prime}$ is a semigroup. Take $i \cdot j=i \wedge j$, for $i, j \in I$. This makes $I$ a finite MTL-algebra (in fact, a Heyting algebra). By

\footnotetext{
${ }^{3}$ In [11 integral ordered commutative monoids are called negative ordered monoids.
} 
regarding $\mathcal{C}_{\left(M_{T}^{\prime}, I\right)}=\left\{M_{i}^{\prime}\right\}_{i \in I}$ and $\mathbf{M}_{\left(M_{T}^{\prime}, I\right)}=\left\{\mu_{i j}: M_{i}^{\prime} \times M_{j}^{\prime} \rightarrow M_{i \wedge j}^{\prime} \mid i, j \in I\right\}$, defined as

$$
\mu_{i j}(a, b)= \begin{cases}a \cdot{ }_{i} b, & \text { if } i=j, \\ a, & \text { if } i<j, \\ b, & \text { if } j<i,\end{cases}
$$

from Lemmas 3.4 and 4.2 the result follows.

\section{REFERENCES}

[1] P. Agliano and F. Montagna, Varieties of BL-algebras. I. General properties, J. Pure Appl. Algebra 181 (2003), no. 2-3, 105-129. MR 1975295

[2] M. Busaniche, Decomposition of BL-chains, Algebra Universalis 52 (2004), no. 4, 519-525 (2005). MR 2120532

[3] J. L. Castiglioni, M. Menni and W. J. Zuluaga Botero, A representation theorem for integral rigs and its applications to residuated lattices, J. Pure Appl. Algebra 220 (2016), no. 10, 3533-3566. MR 3497976.

[4] J. L. Castiglioni and W. J. Zuluaga Botero, On finite MTL-algebras that are representable as poset products of archimedean chains, Fuzzy Sets and Systems 382 (2020), 57-78. MR 4052312

[5] B. A. Davey and H. A. Priestley, Introduction to Lattices and Order, second edition, Cambridge University Press, New York, 2002. MR 1902334

[6] F. Esteva and L. Godo, Monoidal t-norm based logic: towards a logic for left-continuous t-norms, Fuzzy Sets and Systems 124 (2001), no. 3, 271-288. MR 1860848

[7] F. Esteva et al., Hoops and fuzzy logic, J. Logic Comput. 13 (2003), no. 4, 531-555. MR 1999962

[8] S. Jenei and F. Montagna, A proof of standard completeness for Esteva and Godo's logic MTL, Studia Logica 70 (2002), no. 2, 183-192. MR 1894392.

[9] P. Johnstone, A note on the semiabelian variety of Heyting semilattices, in Galois Theory, Hopf Algebras, and Semiabelian Categories, 317-318, Fields Inst. Commun., 43, Amer. Math. Soc., Providence, RI. MR 2075591

[10] C. Noguera, F. Esteva and J. Gispert, On some varieties of MTL-algebras, Log. J. IGPL 13 (2005), no. 4, 443-466. MR 2163142

[11] T. Vetterlein, MTL-algebras arising from partially ordered groups, Fuzzy Sets and Systems 161 (2010), no. 3, 433-443. MR 2566252

\section{J. L. Castiglioni}

CONICET and Departamento de Matemática, Facultad de Ciencias Exactas, Universidad Nacional de La Plata, 50 y 115, 1900 La Plata, Argentina

jlc@mate.unlp.edu.ar

W. J. Zuluaga Botero ${ }^{凶}$

Departamento de Matemática, Facultad de Ciencias Exactas, Universidad Nacional del Centro de la Provincia de Buenos Aires, Pinto 399, 7000 Tandil, Argentina

wijazubo@hotmail.com

Received: October 15, 2019

Accepted: June 16, 2020 